\title{
Lower Bound for RIP Constants and Concentration of Sum of Top Order Statistics
}

\author{
Gen Li, Xingyu Xu, and Yuantao Gu* \\ Manuscript submitted July 5, 2019.
}

\begin{abstract}
Restricted Isometry Property (RIP) is of fundamental importance in the theory of compressed sensing and forms the base of many exact and robust recovery guarantees in this field. Quantitative description of RIP involves bounding the so-called RIP constants of measurement matrices. In this respect, it is noteworthy that most results in literature concerning RIP are upper bounds of RIP constants, which can be interpreted as theoretical guarantee of successful sparse recovery. On the contrary, the land of lower bounds for RIP constants remains uncultivated. Lower bounds of RIP constants, if exist, can be interpreted as the fundamental limit aspect of successful sparse recovery. In this paper, the lower bound of RIP constants Gaussian random matrices are derived, along with a guide for generalization to sub-Gaussian random matrices. This provides a new proof of the fundamental limit that the minimal number of measurements needed to enforce the RIP of order $s$ is $\Omega(s \log (\mathrm{e} N / s))$, which is more straight-forward than the classical Gelfand width argument. Furthermore, in the proof we propose a useful technical tool featuring the concentration phenomenon for top- $k$ sum of a sequence of i.i.d. random variables, which is closely related to mainstream problems in statistics and is of independent interest.
\end{abstract}

Keywords: Lower bound, compressed sensing, order statistics, restricted isometry property, random matrix

\footnotetext{
${ }^{*}$ The authors are with Department of Electronic Engineering, Tsinghua University, Beijing 100084, China. The corresponding author of this paper is Y. Gu (gyt@tsinghua.edu.cn).
} 


\section{Introduction}

Compressed sensing is one of the major achievements in signal processing in the past years. The model of compressed sensing can be typically described as retrieving some data $\mathbf{x} \in$

$\mathbb{R}^{N}$ from linear measurements $\mathbf{y}=\mathbf{A} \mathbf{x}$, where the measurement matrix $\mathbf{A} \in \mathbb{R}^{n \times N}$ is a underdetermined matrix, i.e. $n<N$. Apparently, this task is impossible without proper restrictions on $\mathbf{x}$. The most common restrictions on $\mathbf{x}$ is the sparsity assumption, which requires $\mathbf{x}$ has at most $s$ non-zero entries, where $s \ll N$. If this is the case, $\mathbf{x}$ can be efficiently recovered by numerous algorithms, for example, the so-called $\ell_{1}$-minimization:

$$
\hat{\mathbf{x}}=\arg \min _{\mathbf{z}}\|\mathbf{z}\|_{1} \text { s.t. } \mathbf{y}=\mathbf{A z} \text {. }
$$

Restricted Isometry Property (RIP) has played a dominant role in analysis of such algorithms since it was proposed in [1]. The great power of this concept enables researchers to derive theoretical guarantees for many popular compressed sensing algorithms including $\ell_{1}$-minimization, Orthogonal Matching Pursuit (OMP), Compressive Sampling Matching Pursuit (CoSaMP), Iterative Hard Thresholding (IHT), Hard Thresholding Pursuit (HTP) and so on. Proof of such guarantees is based on the mode of showing or borrowing some upper bounds of the RIP constants of the sensing matrix and then using these upper bounds to analyze specified algorithms. This accounts for the extensive study in the literature on upper bounds of RIP constants. In contrast, few results on the lower bound of RIP constants are known. Similar to the fact that upper bound of RIP constants plays the role of success guarantee for sparse recovery, lower bound of RIP constants could give more insights to the fundamental limit of sparse recovery.

In this paper we give such a lower bound for Gaussian random matrices and provide the method to generalize this result to sub-Gaussian random matrices. We also show that the lower bound is tight by proving a new upper bound of RIP constants, which is a slight improvement on previous results. With this approach we partially rediscover the fact that the minimal number of measurements needed to enforce the RIP of order $s$ is $\Omega(s \log (\mathrm{e} N / s))$. Compared with the classical proof using Gelfand width, our proof bears less generality but is much more straight-forward.

Furthermore, we identify a useful tool among the lines of our proof, which we call by concentration of sum of top order statistics. In the literature of probability and statistics, asymptotic behaviour and concentration phenomenon of order statistics have raised much 
attention [2]. They are also closely related to the study of empirical process, which is one of the mainstream problems in statistics. On the other hand, sum of top order statistics is another classical topic [3] within this field. However, known results on joint and individual concentration of order statistics appear to yield inequalities for sum of top order statistics that are far from optimal. This suggests that more tailored techniques are required to find the optimal bound, as we will do in this paper. To the best of our knowledge, this paper is the first one to establish an exponential concentration inequality for sum of top order statistics. We believe this result is of interest in other fields besides compressed sensing.

We will elucidate the backgrounds and explain our contributions in more technical detail in Section 3 ,

\section{$1.1 \quad$ Notations}

Bold upper case letters, e.g. A, are used to denote matrices, while bold lower case letters, e.g. $\mathbf{x}$, are used to denote vectors. The $s$-th RIP constant of a matrix $\mathbf{A}$, to be defined in Section 2, is denoted by $\delta_{s}(\mathbf{A})$, or simply by $\delta_{s}$ when there is no confusion. $\mathbb{P}(\cdot)$ is the probability of an event. $\mathbb{E}(\cdot)$ and $\mathbb{V}(\cdot)$ denotes respectively the expectation and the variance of a random variable. By convention, a Gaussian matrix is a matrix with i.i.d. standard Gaussian entries. $\Psi_{s}$ denotes the set of non-zero $s$-sparse unit vectors in $\mathbb{R}^{N}$, c.f. Section 2, Denote by $C$ a positive constant that may vary upon each appearance. We write $a(n) \sim b(n)$ if $\lim _{n \rightarrow \infty} a(n) / b(n)=1, a(n) \lesssim b(n)$ if $a(n) \leq C b(n)$ for some constant $C$, and $a(n) \asymp b(n)$ if both $a(n) \lesssim b(n)$ and $b(n) \lesssim a(n)$ are true.

\subsection{Organization}

The rest of this paper is organized as follows. Our main results are presented in Section 2 , where we also demonstrate the important corollary on minimal measurements required for successful recovery. Section 3 is a more involved discussion on backgrounds and motivations of these results. Section 4 [5, and 6 are devoted to the proof of the main results. For simplicity, these results are stated for Gaussian matrices, but most of them can be generalized easily to sub-Gaussian cases. We sketch how such generalization could be done in Section 7. In Section 8, our new bounds are plotted in comparison with previous results. Finally in Section 9, we conclude the paper. 


\section{Main Results}

We begin with necessary definitions. A vector $\mathbf{x}$ is called $s$-sparse, or $\|\mathbf{x}\|_{0} \leq s$, if at most $s$ entries of $\mathbf{x}$ are non-zero. The $s$-th RIP constant of a matrix $\mathbf{A}$ characterizes how close A is to an isometry when restricted to the set of $s$-sparse vectors.

Definition 1 The $s$-th RIP constant $\delta_{s}$ of a matrix $\mathbf{A}$ is defined to be the smallest nonnegative number such that for any s-sparse vector $\mathbf{x}$, the following holds:

$$
\left(1-\delta_{s}\right)\|\mathbf{x}\|^{2} \leq\|\mathbf{A} \mathbf{x}\|^{2} \leq\left(1+\delta_{s}\right)\|\mathbf{x}\|^{2}
$$

RIP constants of random matrices are often asymmetric in the sense that the minimal $\delta$ satisfying $(1-\delta)\|\mathbf{x}\|^{2} \leq\|\mathbf{A} \mathbf{x}\|^{2}$ for all $s$-sparse $\mathbf{x}$ is essentially different from the minimal $\delta$ satisfying $\|\mathbf{A} \mathbf{x}\|^{2} \leq(1+\delta)\|\mathbf{x}\|^{2}$ for all $s$-sparse $\mathbf{x}$. For this reason we need more intricate notations for RIP constants. Denote by $\Psi_{s}$ the set of non-zero unit vectors $\mathbf{x}$ in $\mathbb{R}^{N}$ such that $\|\mathbf{x}\|_{0} \leq s$. Define

$$
\begin{aligned}
& \delta_{s}^{+}=\sup _{\mathbf{x} \in \Psi_{s}}\|\mathbf{A} \mathbf{x}\|^{2}-1, \\
& \delta_{s}^{-}=1-\inf _{\mathbf{x} \in \Psi_{s}}\|\mathbf{A} \mathbf{x}\|^{2} .
\end{aligned}
$$

It is then obvious that

$$
\delta_{s}=\max \left(\delta_{s}^{+}, \delta_{s}^{-}\right)
$$

Hence it suffices to study $\delta_{s}^{+}$and $\delta_{s}^{-}$separately.

The first one of our main results is a lower bound for RIP constants of random matrices. For simplicity, we only state and prove the corresponding results for Gaussian matrices. However, Theorem 1 can be easily generalized to sub-Gaussian distributions, and Proposition 2 is even more universal. Desired readers may find relevant discussions in Section 7.

Theorem 1 Let $\mathbf{A}$ be a Gaussian matrix in $\mathbb{R}^{n \times N}$. Then for some constant $C>0$ and with probability at least $1-C \mathrm{e}^{-n \varepsilon^{2} / C}$, the RIP constants of $\mathbf{\Phi}=\frac{1}{\sqrt{n}} \mathbf{A}$ satisfy

$$
\begin{aligned}
& \delta_{s}^{+} \geq\left((1+\sqrt{p} T)(1-\delta)+\frac{1}{2} p T^{2}\right)^{1 / 2}-1, \\
& \delta_{s}^{-} \geq 1-\left(1-\sqrt{p} T+(1+\sqrt{p} T) \delta+\frac{1}{2} p T^{2}\right)^{1 / 2},
\end{aligned}
$$


where $s / N<1 / 5, p=\frac{s}{n}, \delta=\varepsilon+\left(n \log \frac{N}{s}\right)^{-1 / 2}$, and $T$ is defined as follows: let $Y$ be a $\chi_{1}^{2}$ random variable and $t$ be the $\left(1-\frac{s-1}{N-1}\right)$-quantile of $Y$ (i.e. $\left.\mathbb{P}(Y>t)=\frac{s-1}{N-1}\right)$, then $T=\sqrt{\mathbb{E}(Y \mid Y>t)}$.

The above lower bound is tight in comparison with the following upper bound:

Proposition 1 Let $\mathbf{A}$ be a Gaussian matrix in $\mathbb{R}^{n \times N}$. Then the RIP constants of $\mathbf{\Phi}=\frac{1}{\sqrt{n}} \mathbf{A}$ satisfy

$$
\delta_{s}=\sqrt{p} T+\frac{C}{\sqrt{n \log \frac{N}{s}}}+\frac{1}{2 n}+\varepsilon
$$

with probability at least $1-2 \mathrm{e}^{-n \varepsilon^{2} / 2}$, where $p=\frac{s}{n}<1 / 5$ and $T$ is defined as follows: let $Y$ be a $\chi_{1}^{2}$ random variable and $t$ be the $\left(1-\frac{s}{N}\right)$-quantile of $Y$ (i.e. $\left.\mathbb{P}(Y>t)=\frac{s}{N}\right)$, then $T=\sqrt{\mathbb{E}(Y \mid Y>t)}$.

Remark 1 The upper bound presented is in fact of the same order as the classical one, see Theorem [2. The only improvement is a better multiplicative constant. Such improvement, despite being minor, makes the new upper bound very close to the new lower bound given in Theorem 1, demonstrating the tightness of the lower bound.

The proof of Theorem 1 and Proposition 1 makes crucial use of another main result in this paper, namely the concentration of top sum of order statistics. Recall that for a sequence of i.i.d. random variables $X_{1}, \ldots, X_{n}$, the order statistics $X_{(1)}, \ldots, X_{(n)}$ are its non-increasing rearrangement.

Proposition 2 Let $Y_{1}, \ldots, Y_{n}$ be a sequence of i.i.d. $\chi_{1}^{2}$ random variables, and $Y_{(1)}, \ldots, Y_{(n)}$ be their order statistics. Assume that $k / n<1 / 5$. Define

$$
T_{k}=\sqrt{\frac{1}{k} \sum_{i=1}^{k} Y_{(i)}}
$$

and

$$
T=\sqrt{\mathbb{E}\left(Y_{1} \mid Y_{1}>t\right)}
$$

where $t$ is defined by $\mathbb{P}\left(Y_{1}>t\right)=\frac{k}{n}$, then

$$
\left|\mathbb{E} T_{k}-T\right| \leq \frac{C}{\sqrt{k \log \frac{n}{k}}}
$$

where $C$ is a positive constant, and

$$
\mathbb{P}\left(\left|T_{k}-T\right|>\frac{C}{\sqrt{k \log \frac{n}{k}}}+\varepsilon\right) \leq 2 \mathrm{e}^{-\frac{k \varepsilon^{2}}{2}}, \quad \forall \varepsilon>0 .
$$


We briefly discuss some corollaries of these results, of which the most important one is the minimal number of measurements for successful recovery. First we need an asymptotic estimation of $t$ and $T$ in Theorem 1,

Proposition 3 In the asymptotic regime $N \rightarrow \infty, s / N \rightarrow 0$, we have

$$
t \sim 2 \log \frac{N}{s}, \quad T \sim \sqrt{2 \log \frac{N}{s}} .
$$

As a consequence, in the asymptotic regime $N \rightarrow \infty, s / N \leq \gamma$ for some constant $\gamma \in$ $(0,1 / 5)$, we have

$$
t \asymp \log \frac{N}{s}, \quad T \asymp \sqrt{\log \frac{N}{s}} .
$$

Proof See Section 10.

Corollary 1 (Minimal number of measurements) The minimal number of random Gaussian measurements to enforce $R I P$ is $\Omega(s \log (N / s))$.

Proof By Theorem 1, the RIP is enforced only if $p T^{2}$ is bounded by some constant. From the foregoing asymptotic analysis we see that $T \asymp \sqrt{\log \frac{N}{s}}$. Thus $n=s / p=s T^{2} /\left(p T^{2}\right)=$ $\Omega\left(s \log \frac{N}{s}\right)$.

\section{Related Works}

\subsection{Restricted Isometry Property}

In the literature of compressed sensing, RIP is a powerful tool to prove exact and robust recovery results for various algorithms. Here exact recovery means that the algorithm recovers $\mathbf{x}$ exactly from the measurements $\mathbf{y}=\mathbf{A x}$ for all $s$-sparse $\mathbf{x}$, and robust recovery means that the algorithm returns from noisy measurements $\mathbf{y}=\mathbf{A x}+\mathbf{e}$ an estimate $\tilde{\mathbf{x}}$ of $\mathbf{x}$ with accuracy

$$
\|\mathbf{x}-\tilde{\mathbf{x}}\|_{2} \leq C_{1} \frac{\sigma_{s}(\mathbf{x})_{1}}{\sqrt{s}}+C_{2} \tau
$$

for all vectors $\mathbf{x}$, where $C_{1}, C_{2}$ are positive constants, $\sigma_{s}(\mathbf{x})_{p}$ is the $\ell_{p}$-compressibility of $\mathbf{x}$, defined as

$$
\sigma_{s}(\mathbf{x})_{p}=\inf _{\|\mathbf{z}\|_{0} \leq s}\|\mathbf{x}-\mathbf{z}\|_{p}
$$

and $\tau$ is a deterministic upper bound of the noise level satisfying $\tau \geq\|\mathbf{e}\|_{2}$. We partly borrow from [4] the following Table 1 on the state-of-art results of RIP requirements for 
Table 1: Requirements on RIP constants for various recovery algorithms [4].

\begin{tabular}{cc} 
Algorithm & Requirements \\
\hline$\ell_{1}$-minimization & $\delta_{s}<\frac{1}{3}$ or $\delta_{2 s}<\frac{4}{\sqrt{41}}$ \\
OMP & $\delta_{s}<\frac{1}{1+\sqrt{s}}$ or $\delta_{13 s}<\frac{1}{6}$ \\
CoSaMP & $\delta_{4 s} \leq \frac{\sqrt{\sqrt{11 / 3}-1}}{2}$ \\
IHT, HTP & $\delta_{3 s}<\frac{1}{\sqrt{3}}$ \\
\hline
\end{tabular}

various algorithms to ensure successful exact and robust recovery for $s$-sparse vectors. All of these results can be found, for instance, in [5] and the references therein.

Ideally, if one may effectively evaluate the RIP constants of a given matrix $\mathbf{A}$, not much is left to worry about: with the precise value of RIP constants it can be inferred from Table1 whether we have theoretical guarantee for any of our favourite algorithms. Unfortunately, this is impossible due to the NP-hardness of evaluating RIP constants [6]. In fact, even qualitatively certifying RIP is NP-hard [7]. A common substitute for the precise values of RIP in theoretical anlysis is the upper bound of RIP constants, which would suffice to guarantee successful recovery. The RIP upper bound for sub-Gaussian matrices, stated as below, is a landmark of such results.

Theorem 2 [8] Let $\mathbf{A}$ be a $n \times N$ random matrix with i.i.d. (centered) sub-Gaussian entries of variance 1 and sub-Gaussian norm uniformly bounded by $K>0$. Then the RIP constants of $\boldsymbol{\Phi} \triangleq \frac{1}{\sqrt{n}} \mathbf{A}$ satisfies

$$
\mathbb{P}\left(\delta_{s}>\delta\right) \leq 2\left(\begin{array}{c}
N \\
s
\end{array}\right) \exp \left(-c_{1} \delta^{2} n+c_{2} s\right)
$$

where $c_{1}, c_{2}$ are some positive constants that depend only on $K$.

Corollary 2 For measurement matrix $\mathbf{\Phi}$ as in Theorem 2 , there exists some positive constant $C$ that depends only on $K$, such that $\delta_{s} \leq \delta$ holds with probability at least $1-\varepsilon$ whenever

$$
n \geq C \delta^{-2}\left(s \log (\mathrm{e} N / s)+\log \left(2 \varepsilon^{-1}\right)\right)
$$

The above corollary indicates that when the number of measurements $n \gtrsim s \log (e N / s)$, successful recovery is guaranteed with high (in fact, $1-2 \exp \left(-C^{\prime} \delta^{-2} m\right)$ ) probability. 
We turn to the opposite side of the problem: what is the minimal number of measurements when successful recovery is to be expected? A classical argument by estimating Gelfand width [9] solves this problem by showing that for any measurement matrix $\mathbf{A} \in \mathbb{R}^{n \times N}$ and any recovery algorithm $\Delta$, interpreted as a map $\mathbb{R}^{n} \rightarrow \mathbb{R}^{N}$, if $\|\mathbf{x}-\Delta(\mathbf{A x})\|_{2} \leq C \sigma_{s}(\mathbf{x})_{1}$ holds for all $\mathbf{x}$, we necessarily have

$$
n \gtrsim s \log \left(\frac{\mathrm{e} N}{s}\right)
$$

This, when combined with Table 1, yields the following corollary (in a stronger form).

Corollary 3 If the $s$-th RIP constant of $\mathbf{A} \in \mathbb{R}^{n \times N}$ satisfies $\delta_{s}<\frac{1}{\sqrt{3}}$, then

$$
n \geq c s \log \left(\frac{\mathrm{e} N}{s}\right)
$$

for some constant $c>0$ depending only on $\delta_{s}$.

Taking $\varepsilon=1 / 2$ in (10) and comparing (10) and (11), one readily checks that the bound in (10) is optimal up to a multiplicative constant. These pieces when put together constitutes an almost-conclusive answer to the problem of minimal number of measurements required for successful recovery. However, two issues remain unsolved: i) Theorem 2 is a standard application of random matrix theory, which is a tool intimate for compressed sensing society, while the proof using Gelfand width is indirect and even involves analysis of $\ell_{1}$-minimization algorithm; ii) (9) gives a probabilistic bound of RIP constants, while (11) is deterministic. We therefore pose the following questions:

- Is there a more direct (i.e. random-matrix-theoretic) proof of (111)?

- Can we find a probabilistic lower bound of RIP constants, possibly by bounding the probability $\mathbb{P}\left(\delta_{s} \leq \delta\right)$ for small $\delta$ ?

Theorem 1 is an affirmative answer to these questions.

\subsection{Concentration of Order Statistics}

Distribution of order statistics is a well-investigated topic; the well-known Rényi's representation provides an explicit formula for their distribution function [3]. On the other hand, concentration of order statistics is still an active field of research [2]. Many researches in this vein were inspired by the concentration of measure phemonenon [10]. However, general 
principles in concentration of measure theory do not supply satisfactory bounds for order statistics. For example, it is known that $\mathbb{V}\left(X_{(\lfloor n / 2\rfloor)}\right)=O(1 / n)$, while the powerful logarithmic Sobolev inequality in concentration of measure theory implies only $\mathbb{V}\left(X_{(\lfloor n / 2\rfloor)}\right) \leq 1$. Additional efforts are in need to establish tight bounds for concentration of order statistics.

The most notable results on concentration of order statistics are a series of inequalities stemming from Glivenko-Cantelli theorem. We record here one of such inequalities for reference.

Theorem 3 (Dvoretzky-Kiefer-Wolfowitz inequality1, [11]) Let $X_{1}, \ldots, X_{n}$ be i.i.d. random variables with cumulative distribution function $F(x)$. Denote by $F_{n}$ the associated empirical distribution function, defined by

$$
F_{n}(x)=\frac{1}{n} \sum_{i=1}^{n} \mathbf{1}_{\left\{X_{i} \leq x\right\}} .
$$

Then for any $\varepsilon>0$ the following holds:

$$
\mathbb{P}\left(\sup _{x \in \mathbb{R}}\left|F_{n}(x)-F(x)\right|>\varepsilon\right) \leq 2 \exp \left(-2 n \varepsilon^{2}\right) .
$$

In probability theory, quantities in the form of $\mathbf{1}_{\{X \leq x\}}$ are (heuristically) considered to be of zero-th order, in contrast with first (second, third, ...) order quantities such as $X\left(X^{2}, X^{3}, \ldots\right)$. One may thus regard Theorem 3 as a zero-th order joint concentration theorem for order statistics. Concentration theorem in higher order for any individual of order statistics is also available in the literature, c.f. [2], but is described in a rather complicated form which does not imply convenient tail bound. On the other hand, first order joint concentration theorem, i.e. a tail bound on concentration of $\sum_{i=1}^{k} X_{(i)}$, is almost unknown. Our result may hopefully fill this vacancy.

\section{Proof of Theorem 1}

For convenience we first set up some notations. Let

$$
\begin{aligned}
\sigma_{\max }^{s}(\mathbf{A}) & =\sup _{\mathbf{x} \in \Psi_{s}}\|\mathbf{A} \mathbf{x}\|, \\
\sigma_{\min }^{s}(\mathbf{A}) & =\inf _{\mathbf{x} \in \Psi_{s}}\|\mathbf{A} \mathbf{x}\| .
\end{aligned}
$$

\footnotetext{
${ }^{1}$ A weaker form of this theorem is well-known to statisticians as Kolmogorov-Smirnov test.
} 
By definition we have

$$
\begin{aligned}
& \delta_{s}^{+}(\boldsymbol{\Phi})=\frac{1}{\sqrt{n}} \sigma_{\max }^{s}(\mathbf{A})-1, \\
& \delta_{s}^{+}(\boldsymbol{\Phi})=1-\frac{1}{\sqrt{n}} \sigma_{\min }^{s}(\mathbf{A}) .
\end{aligned}
$$

In this section we assume that $\mathbf{A}$ is a random matrix with i.i.d. standard Gaussian entries. For an $N$-dimensional unit vector $\mathbf{v}=\left(v_{1}, v_{2}, \ldots, v_{N}\right)$, we have

$$
\|\mathbf{A} \mathbf{v}\|^{2}=v_{1}^{2}\left\|\mathbf{a}_{1}\right\|^{2}+2 v_{1} \sum_{i=2}^{N} v_{i}\left\langle\mathbf{a}_{1}, \mathbf{a}_{i}\right\rangle+\sum_{i, j=2}^{N} v_{i} v_{j}\left\langle\mathbf{a}_{i}, \mathbf{a}_{j}\right\rangle .
$$

Set

$$
\begin{aligned}
x_{i} & =\frac{\left\langle\mathbf{a}_{1}, \mathbf{a}_{i}\right\rangle}{\left\|\mathbf{a}_{1}\right\|}, \\
\mathbf{b}_{i} & =\mathbf{a}_{i}-x_{i} \frac{\mathbf{a}_{1}}{\left\|\mathbf{a}_{1}\right\|},
\end{aligned}
$$

for $i=2, \ldots, N$. By a well-known property of Gaussian distribution, $\left\{\mathbf{a}_{1}, x_{2}, \ldots, x_{N}\right\}$ are jointly independent and are all standard Gaussian vectors/variables. Conditioning on $\mathbf{a}_{1}$, we see that

$$
\left\{x_{2}, \ldots, x_{N}, \mathbf{b}_{2}, \ldots, \mathbf{b}_{N}\right\}
$$

are jointly independent, and $\mathbf{b}_{i}$ 's are Gaussian vectors with covariance matrix

$$
\boldsymbol{\Sigma}=\mathbf{I}-\frac{\mathbf{a}_{1} \mathbf{a}_{1}^{\mathrm{T}}}{\left\|\mathbf{a}_{1}\right\|^{2}}
$$

With (15) and the fact that $\left\langle\mathbf{a}_{1}, \mathbf{b}_{i}\right\rangle=0$, (14) can be expressed as

$$
\begin{aligned}
\|\mathbf{A} \mathbf{v}\|^{2} & =v_{1}^{2}\left\|\mathbf{a}_{1}\right\|^{2}+2 v_{1}\left\|\mathbf{a}_{1}\right\| \sum_{i=2}^{N} v_{i} x_{i}+\sum_{i, j=2}^{N} v_{i} v_{j}\left(x_{i} x_{j}+\left\langle\mathbf{b}_{i}, \mathbf{b}_{j}\right\rangle\right) \\
& =v_{1}^{2}\left\|\mathbf{a}_{1}\right\|^{2}+2 v_{1}\left\|\mathbf{a}_{1}\right\| \sum_{i=2}^{N} v_{i} x_{i}+\left(\sum_{i=2}^{N} v_{i} x_{i}\right)^{2}+\sum_{i, j=2}^{N} v_{i} v_{j}\left\langle\mathbf{b}_{i}, \mathbf{b}_{j}\right\rangle .
\end{aligned}
$$

We are interested in extremal values of $\|\mathbf{A v}\|^{2}$ as $\mathbf{v}$ ranges over the set of $s$-sparse unit vectors. To establish the lower bound in Theorem 1, we will designate some specific values of $\mathbf{v}$ to estimate these extremal values. That is, for any specific choice of $s$-sparse unit vectors $\mathbf{v}^{\prime}$, we have

$$
\left(\sigma_{\max }^{s}(\mathbf{A})\right)^{2} \geq\left\|\mathbf{A} \mathbf{v}^{\prime}\right\|^{2}
$$

and

$$
\left(\sigma_{\min }^{s}(\mathbf{A})\right)^{2} \leq\left\|\mathbf{A} \mathbf{v}^{\prime}\right\|^{2} .
$$


In case that $\left\|\mathbf{A} \mathbf{v}^{\prime}\right\|^{2}$ is sufficiently close to $\max _{\mathbf{v} \in \Psi_{s}}\|\mathbf{A v}\|^{2}$ (resp. $\min _{\mathbf{v} \in \Psi_{s}}\|\mathbf{A} \mathbf{v}\|^{2}$ ) and is easy to compute, the above method will provide a satisfactory lower bound for $\max _{\mathbf{v} \in \Psi_{s}}\|\mathbf{A v}\|^{2}$ $\left(\right.$ resp. $\left.\min _{\mathbf{v} \in \Psi_{s}}\|\mathbf{A} \mathbf{v}\|^{2}\right)$.

Next we show how to construct such $\mathbf{v}^{\prime}$. Take $v_{1}^{\prime}=1 / \sqrt{2}$. By the proof of CauchySchwarz inequality, there exists a suitable choice of $\left(v_{2}^{\prime}, \ldots, v_{N}^{\prime}\right)$ which is $(s-1)$-sparse and fulfills

$$
\begin{aligned}
\sum_{i=2}^{N} v_{i}^{\prime 2} & =\frac{1}{2} \\
\sum_{i=2}^{N} v_{i}^{\prime} x_{i} & =\sqrt{\sum_{i=2}^{N} v_{i}^{\prime 2}} \sqrt{\sum_{j=1}^{s-1} x_{(j)}^{2}} .
\end{aligned}
$$

Moreover, such choice makes $\mathbf{v}^{\prime}$ a $\left(x_{2}, \ldots, x_{N}\right)$-measurable random vector. Combining the above equations, we have

$$
\left\|\mathbf{A} \mathbf{v}^{\prime}\right\|^{2}=\frac{1}{2}\left\|\mathbf{a}_{1}\right\|^{2}+\left\|\mathbf{a}_{1}\right\| \sqrt{\sum_{j=1}^{s-1} x_{(j)}^{2}}+\frac{1}{2} \sum_{j=1}^{s-1} x_{(j)}^{2}+\sum_{i, j=2}^{N} v_{i}^{\prime} v_{j}^{\prime}\left\langle\mathbf{b}_{i}, \mathbf{b}_{j}\right\rangle .
$$

On the other hand, taking

$$
\mathbf{v}^{\prime \prime}=\left[v_{1}^{\prime},-v_{2}^{\prime},-v_{3}^{\prime}, \ldots,-v_{N}^{\prime}\right]^{\mathrm{T}},
$$

we have

$$
\left\|\mathbf{A} \mathbf{v}^{\prime \prime}\right\|^{2}=\frac{1}{2}\left\|\mathbf{a}_{1}\right\|^{2}-\left\|\mathbf{a}_{1}\right\| \sqrt{\sum_{j=1}^{s-1} x_{(j)}^{2}}+\frac{1}{2} \sum_{j=1}^{s-1} x_{(j)}^{2}+\sum_{i, j=2}^{N} v_{i}^{\prime} v_{j}^{\prime}\left\langle\mathbf{b}_{i}, \mathbf{b}_{j}\right\rangle .
$$

A lower bound (resp. upper bound) of $\left\|\mathbf{A} \mathbf{v}^{\prime}\right\|^{2}$ (resp. $\left\|\mathbf{A} \mathbf{v}^{\prime \prime}\right\|^{2}$ ), hence of $\max _{\mathbf{v} \in \Psi_{s}}\|\mathbf{A} \mathbf{v}\|^{2}$ (resp. $\min _{\mathbf{v} \in \Psi_{s}}\|\mathbf{A v}\|^{2}$ ), is obtained immediately as a consequence of Proposition 2 and standard concentration inequalities for Gaussian quadratic forms. In fact, from Bernstein inequality we see that for some universal constant $C>0$ and any $\varepsilon>0$, the following holds:

$$
\mathbb{P}\left(\frac{1}{n}\left|\left\|\mathbf{a}_{1}\right\|^{2}-n\right|>\varepsilon\right) \leq 2 \exp \left(-C n \min \left(\varepsilon, \varepsilon^{2}\right)\right)
$$

Similarly, setting

$$
\Gamma_{\varepsilon} \triangleq\left\{\frac{1}{n}\left|\sum_{i, j=2}^{N} v_{i}^{\prime} v_{j}^{\prime}\left\langle\mathbf{b}_{i}, \mathbf{b}_{j}\right\rangle-(n-1)\left(1-v_{1}^{2}\right)\right|>\varepsilon\right\},
$$


and by conditional independence of $\mathbf{v}^{\prime}$ and $\mathbf{b}_{i}$, we have

$$
\begin{aligned}
\mathbb{P}\left(\Gamma_{\varepsilon} \mid \mathbf{a}, v_{1}^{\prime}, \ldots, v_{n}^{\prime}\right) & \leq 2 \exp \left[-C n \min \left(\frac{\varepsilon}{1-v_{1}^{2}}, \frac{\varepsilon^{2}}{\left(1-v_{1}^{2}\right)^{2}}\right)\right] \\
& \leq 2 \exp \left(-C n \min \left(\varepsilon, \varepsilon^{2}\right)\right),
\end{aligned}
$$

which follows from Hanson-Wright inequality [12]. Thus by integrating we obtain

$$
\mathbb{P}\left(\Gamma_{\varepsilon}\right) \leq 2 \exp \left(-C n \min \left(\varepsilon, \varepsilon^{2}\right)\right)
$$

Applying Proposition 2 to $\sum_{j=1}^{s-1} x_{(j)}^{2}$ and taking into account the foregoing arguments, the conclusion of Theorem 1 follows immediately.

\section{Proof of Proposition 1}

The proof is a slight modification of the standard one. From the definition of $\sigma_{\max }^{s}$ and $\sigma_{\min }^{s}$, it is readily verified that

Proposition $4 \sigma_{\max }^{s}(\mathbf{A}), \sigma_{\min }^{s}(\mathbf{A})$ is 1-Lipschitz in $\mathbf{A}$. In other words, for $\mathbf{A}_{1}, \mathbf{A}_{2} \in \mathbb{R}^{n \times N}$, we have

$$
\begin{gathered}
\sigma_{\max }^{s}\left(\mathbf{A}_{1}\right)-\sigma_{\max }^{s}\left(\mathbf{A}_{2}\right) \leq\left\|\mathbf{A}_{1}-\mathbf{A}_{2}\right\|_{\mathrm{F}} \\
\sigma_{\min }^{s}\left(\mathbf{A}_{1}\right)-\sigma_{\min }^{s}\left(\mathbf{A}_{2}\right) \leq\left\|\mathbf{A}_{1}-\mathbf{A}_{2}\right\|_{\mathrm{F}}
\end{gathered}
$$

where $\|\cdot\|_{\mathrm{F}}$ denotes the Frobenius norm. In fact, (22) and (23) hold even if Frobenius norm is replaced by operator norm.

By Proposition 4 and concentration of measure (Appendix 11), we have

$$
\begin{aligned}
& \mathbb{P}\left(\sigma_{\text {max }}^{s}(\mathbf{A})>\mathbb{E} \sigma_{\text {max }}^{s}(\mathbf{A})+\varepsilon\right) \leq \mathrm{e}^{-\frac{\varepsilon^{2}}{2}}, \\
& \mathbb{P}\left(\sigma_{\text {min }}^{s}(\mathbf{A})<\mathbb{E} \sigma_{\text {min }}^{s}(\mathbf{A})-\varepsilon\right) \leq \mathrm{e}^{-\frac{\varepsilon^{2}}{2}}
\end{aligned}
$$

To prove Proposition 11, it suffices to bound $\mathbb{E} \sigma_{\max }^{s}(\mathbf{A})$ and $\mathbb{E} \sigma_{\min }^{s}(\mathbf{A})$. For such purpose we need a well-known comparison lemma for Gaussian process depicted in Appendix 11, Lemma 3, Specifically, we shall use the following consequence of Lemma 3 .

Lemma 1 Let $\mathbf{g}, \mathbf{h}$ be standard Gaussian random vectors. Define two Gaussian processes $X_{\mathbf{u}, \mathbf{v}}$ and $Y_{\mathbf{u}, \mathbf{v}}$ on the set $\left\{\mathbf{u} \in \mathbb{S}^{n-1}, \mathbf{v} \in \Psi_{k}\right\}$ as following:

$$
\begin{aligned}
X_{\mathbf{u}, \mathbf{v}} & =\langle\mathbf{A u}, \mathbf{v}\rangle, \\
Y_{\mathbf{u}, \mathbf{v}} & =\langle\mathbf{g}, \mathbf{u}\rangle+\langle\mathbf{h}, \mathbf{v}\rangle,
\end{aligned}
$$


then we have

$$
\begin{aligned}
& \mathbb{E} \max _{\mathbf{v} \in \Psi_{k}} \max _{\mathbf{u} \in \mathbb{S}^{n-1}} X_{\mathbf{u}, \mathbf{v}} \leq \mathbb{E} \max _{\mathbf{v} \in \Psi_{k}} \max _{\mathbf{u} \in \mathbb{S}^{n-1}} Y_{\mathbf{u}, \mathbf{v}}, \\
& \mathbb{E} \min _{\mathbf{v} \in \Psi_{k}} \max _{\mathbf{u} \in \mathbb{S}^{n-1}} X_{\mathbf{u}, \mathbf{v}} \geq \mathbb{E} \min _{\mathbf{v} \in \Psi_{k}} \max _{\mathbf{u} \in \mathbb{S}^{n-1}} Y_{\mathbf{u}, \mathbf{v}} .
\end{aligned}
$$

Proof See Section 11.

\subsection{Upper Bound of $\mathbb{E} \sigma_{\max }^{s}(\mathrm{~A})$}

By Lemma 1, we have

$$
\begin{aligned}
\mathbb{E} \sigma_{\max }^{s}(\mathbf{A}) & =\mathbb{E} \max _{\mathbf{v} \in \Psi_{s}} \max _{\mathbf{u} \in \mathbb{S}^{n-1}} \mathbf{u}^{\mathrm{T}} \mathbf{A} \mathbf{v} \\
& \leq \mathbb{E} \max _{\mathbf{u} \in \mathbb{S}^{n-1}}\langle\mathbf{g}, \mathbf{u}\rangle+\mathbb{E} \max _{\mathbf{v} \in \Psi_{s}}\langle\mathbf{h}, \mathbf{v}\rangle \\
& =\mathbb{E}\|\mathbf{g}\|+\mathbb{E}\left\|\mathbf{h}_{k}^{\sharp}\right\|
\end{aligned}
$$

where

$$
\mathbf{h}_{k}^{\sharp}=\left[h_{(1)}, \ldots, h_{(k)}, 0, \ldots, 0\right]^{\mathrm{T}} \in \mathbb{R}^{N}
$$

is a $k$-sparse vector obtained from $\mathbf{h}$ by expunging all entries of $\mathbf{h}$ except those $k$ entries with largest magnitudes. Here $h_{(i)}$ denotes the entry in $\mathbf{h}$ with $i$-th largest magnitude. Note that $h_{(i)}^{2}$ can be viewed as the $i$-th order statistic of i.i.d. $\chi_{1}^{2}$ random variables $h_{1}^{2}, \ldots, h_{n}^{2}$. Thus

$$
\mathbb{E}\left\|\mathbf{h}_{k}^{\sharp}\right\|=\mathbb{E} \sqrt{\sum_{i=1}^{k} h_{(i)}^{2}}
$$

can be bounded with Proposition 2, We have

$$
\mathbb{E} \sqrt{\sum_{i=1}^{k} h_{(i)}^{2}} \leq \sqrt{k} T+\frac{C}{\sqrt{\log \frac{N}{k}}} .
$$

On the other hand, it is well-known that

$$
\mathbb{E}\|\mathbf{g}\|=\frac{\sqrt{2} \Gamma\left(\frac{n+1}{2}\right)}{\Gamma\left(\frac{n}{2}\right)},
$$

and by Kazarinoff's inequality for binomial coefficients [13] (or simply by Cauchy-Schwarz) we have

$$
\mathbb{E}\|\mathbf{g}\| \leq \sqrt{n}
$$

Thus

$$
\mathbb{E} \sigma_{\max }^{s}(\mathbf{A}) \leq \sqrt{n}+\sqrt{k} T+\frac{C}{\sqrt{\log \frac{N}{k}}}
$$




\subsection{Lower Bound of $\mathbb{E} \sigma_{\min }^{s}(\mathbf{A})$}

Similar to (26), one may invoke Lemma 1 to obtain

$$
\begin{aligned}
\mathbb{E} \sigma_{\min }^{s}(\mathbf{A}) & =\mathbb{E} \min _{\mathbf{v} \in \Psi_{s}} \max _{\mathbf{u} \in \mathbb{S}^{n-1}} \mathbf{u}^{\mathrm{T}} \mathbf{A} \mathbf{v} \\
& \geq \mathbb{E} \max _{\mathbf{u} \in \mathbb{S}^{n-1}}\langle\mathbf{g}, \mathbf{u}\rangle+\mathbb{E} \min _{\mathbf{v} \in \Psi_{s}}\langle\mathbf{h}, \mathbf{v}\rangle \\
& =\mathbb{E}\|\mathbf{g}\|-\mathbb{E}\left\|\mathbf{h}_{k}^{\sharp}\right\| .
\end{aligned}
$$

Now by Kazarinoff's inequality we have

$$
\mathbb{E}\|\mathbf{g}\| \geq \sqrt{n-\frac{1}{2}}
$$

Combining (27), (31), and (32), we have

$$
\mathbb{E} \sigma_{\min }^{s}(\mathbf{A}) \geq \sqrt{n-\frac{1}{2}}-\sqrt{k} T-\frac{C}{\sqrt{\log \frac{N}{k}}} .
$$

Note that $\sqrt{n-\frac{1}{2}} \geq \sqrt{n}-\frac{1}{2 \sqrt{n}}$. The conclusion of Proposition 1 then follows from (12), (13), and (24), (25), (30), (33).

\section{Concentration of order statistics}

In this section we will prove Proposition 2, The quantity of interest here is the sum $\sum_{i=1}^{k} X_{(i)}$, where $X_{(1)} \geq \ldots \geq X_{(n)}$ are the order statistics of i.i.d. non-negative random variables $X_{1}, \ldots, X_{n}$. We begin with a concentration inequality for $X_{(k)}$ which can be regarded as a local version of Theorem 3 .

Lemma 2 Let $X_{1}, \ldots, X_{n}$ be a sequence of i.i.d. non-negative random variables with distribution function $F$, and $X_{(1)}, \ldots, X_{(n)}$ be theirs order statistics. Denote $\alpha=k / n<1 / 2$. Assume $t^{-}, t^{+}$are positive real numbers such that

$$
\begin{aligned}
& \mathbb{P}\left(X_{1}>t^{-}\right)=\alpha+\delta, \\
& \mathbb{P}\left(X_{1}>t^{+}\right)=\alpha-\delta,
\end{aligned}
$$

where $\delta$ is a small positive constant, e.g. $\delta \in(1-\alpha, \alpha)$. Then

$$
\begin{aligned}
& \mathbb{P}\left(X_{(k+1)} \leq t^{-}\right) \leq \exp \left(-n \delta^{2}\left(\frac{1}{\alpha}+\frac{1}{1-\alpha}\right) \log \frac{\mathrm{e}}{2}\right), \\
& \mathbb{P}\left(X_{(k-1)}>t^{+}\right) \leq \exp \left(-n \delta^{2}\left(\frac{1}{\alpha}+\frac{1}{1-\alpha}\right) \log \frac{\mathrm{e}}{2}\right) .
\end{aligned}
$$


In particular, since $X_{(k+1)} \leq X_{(k)} \leq X_{(k-1)}$, we have

$$
\mathbb{P}\left(t^{-}<X_{(k)} \leq t^{+}\right) \geq 1-2 \exp \left(-n \delta^{2}\left(\frac{1}{\alpha}+\frac{1}{1-\alpha}\right) \log \frac{\mathrm{e}}{2}\right) .
$$

Proof Observe that $X_{(k+1)} \leq t^{-}$is equivalent to $\sum_{i=1}^{n} \mathbf{1}_{\left\{X_{i}>t^{-}\right\}} \leq k$. Since $\mathbf{1}_{\left\{X_{i}>t^{-}\right\}}$are i.i.d. Bernoulli variables with

$$
\begin{aligned}
\mathbb{P}\left(\mathbf{1}_{\left\{X_{i}>t^{-}\right\}}=1\right) & =\mathbb{P}\left(X_{i}>t^{-}\right) \\
& =\alpha+\delta
\end{aligned}
$$

we see that $\sum_{i=1}^{n} \mathbf{1}_{\left\{X_{i}>t^{-}\right\}}$follows binomial distribution $B(n, \alpha+\delta)$. Thus one may apply classical entropy-type tail bounds (see [14] for example) to obtain

$$
\mathbb{P}\left(\sum_{i=1}^{n} \mathbf{1}_{\left\{X_{i}>t^{-}\right\}} \leq k\right) \leq \exp (-n D(\alpha \| \alpha+\delta)),
$$

where $D(\alpha \| \alpha+\delta)$ is the relative entropy given by

$$
D(\alpha \| \alpha+\delta)=\alpha \log \frac{\alpha}{\alpha+\delta}+(1-\alpha) \log \frac{1-\alpha}{1-\alpha-\delta} .
$$

Equivalently, we have

$$
D(\alpha \| \alpha+\delta)=-\alpha \log \left(1+\frac{\delta}{\alpha}\right)-(1-\alpha) \log \left(1-\frac{\delta}{1-\alpha}\right) .
$$

For $x \in(-1,1)$, we have the following elementary inequality:

$$
\log (1+x) \leq x-\left(\log \frac{\mathrm{e}}{2}\right) x^{2}
$$

hence

$$
D(\alpha \| \alpha+\delta) \geq \delta^{2}\left(\frac{1}{\alpha}+\frac{1}{1-\alpha}\right) \log \frac{\mathrm{e}}{2} .
$$

Plugging (37) into (36) completes the proof of (34). The proof of (35) is verbatim.

Remark 2 The quantile $t^{-}$and $t^{+}$in Lemma 2 does exist for any (reasonable) $\delta$ when the distribution $F$ is continous on $[0, \infty)$. Assume further that $F$ is absolutely continous on $[0, \infty)$ and $f$ is its density, then

$$
\mathbb{P}\left(\left|X_{(k)}-t\right|>\varepsilon\right) \leq 2 \exp \left(-K n \delta^{2}\left(\alpha^{-1}+(1-\alpha)^{-1}\right)\right)
$$

where $t$ is such that

$$
\mathbb{P}\left(X_{1}>t\right)=1-\alpha
$$

and

$$
K=\left(\log \frac{\mathrm{e}}{2}\right) \inf _{|x-t| \leq \varepsilon} f^{2}(x)
$$


The proof proceeds as following. Note that

$$
\left|T_{k}-T\right| \leq\left|T_{k}-\mathbb{E} T_{k}\right|+\left|\mathbb{E} T_{k}-\sqrt{\mathbb{E} T_{k}^{2}}\right|+\left|\sqrt{\mathbb{E} T_{k}^{2}}-T\right|
$$

We will analyze the three terms in the right hand side to prove the required concentration inequality for $\left|T_{k}-T\right|$. The bound of $\left|\mathbb{E} T_{k}-T\right|$ is obtained as a byproduct.

It follows from rearrangement inequality that $T_{k}$ is a $\sqrt{\frac{1}{k}}$-Lipschitz function in $\mathbf{X}=$ $\left(X_{1}, \ldots, X_{n}\right)$. By concentration of measure (Appendix 11), we have

$$
\mathbb{P}\left(\left|T_{k}-\mathbb{E} T_{k}\right|>\varepsilon\right) \leq 2 \mathrm{e}^{-\frac{k \varepsilon^{2}}{2}}, \quad \forall \varepsilon>0
$$

and consequently

$$
\mathbb{P}\left(\left|T_{k}^{2}-\left(\mathbb{E} T_{k}\right)^{2}\right|>t^{2}+2 t \mathbb{E} T_{k}\right) \leq 2 \mathrm{e}^{-\frac{k t^{2}}{2}} .
$$

To bound $\mathbb{E} T_{k}-\sqrt{\mathbb{E} T_{k}^{2}}$, we instead inspect $\mathbb{E} T_{k}^{2}-\left(\mathbb{E} T_{k}\right)^{2}$. Let

$$
f(x)=\frac{1}{\sqrt{2 \pi x}} \mathrm{e}^{-\frac{x}{2}}
$$

and $F(x)$ be the p.d.f. and the c.d.f. of $\chi_{1}^{2}$ distribution respectively.

Observe that

$$
\begin{aligned}
\left|\mathbb{E} T_{k}^{2}-\left(\mathbb{E} T_{k}\right)^{2}\right| & \leq \mathbb{E}\left|T_{k}^{2}-\left(\mathbb{E} T_{k}\right)^{2}\right| \\
& =\int_{0}^{\infty} \mathbb{P}\left(\left|T_{k}^{2}-\left(\mathbb{E} T_{k}\right)^{2}\right|>x\right) \mathrm{d} x \\
& \leq \int_{0}^{\infty} \mathbb{P}\left(\left|T_{k}^{2}-\left(\mathbb{E} T_{k}\right)^{2}\right|>x\right) \mathrm{d} x \\
& \leq \int_{0}^{\infty} 2 \mathrm{e}^{-\frac{k x^{2}}{2}} \mathrm{~d}\left(x^{2}+2 x \mathbb{E} T_{k}\right) \\
& =\frac{4}{k}+\frac{2 \sqrt{2 \pi} \mathbb{E} T_{k}}{\sqrt{k}} .
\end{aligned}
$$

Next, we bound the difference between $\mathbb{E} T_{k}^{2}$ and $T^{2}$.

Given $X_{(k+1)}^{2}, T_{k}^{2}$ can be seen as a function of $k$ i.i.d. random variable $Y_{1}, \ldots, Y_{k}$ with $Y_{i} \sim X^{2} \mid X^{2}>X_{(k+1)}^{2}$, where $T_{k}^{2}=\frac{1}{k} \sum_{i=1}^{k} Y_{k}$ and $X^{2} \sim \chi_{1}^{2}$, then the conditional expectation of $T_{k}^{2}$ is

$$
\begin{aligned}
\mathbb{E}\left(T_{k}^{2} \mid X_{(k+1)}^{2}\right) & =\mathbb{E} \frac{1}{k} \sum_{i=1}^{k} Y_{i} \\
& =\mathbb{E} Y_{1}
\end{aligned}
$$


We inspect the quantity $\mathbb{E}\left(X^{2} \mid X^{2}>s\right)$. By straight-forward computation, we have

$$
\begin{aligned}
\mathbb{E}\left(X^{2} \mid X^{2}>s\right) & =\frac{\int_{s}^{\infty} x f(x) \mathrm{d} x}{\int_{s}^{\infty} f(x) \mathrm{d} x} \\
& =\frac{s}{\mathbb{E} \sqrt{\frac{s}{s+Y}}}+1,
\end{aligned}
$$

where $Y \sim \operatorname{Exp}\left(\frac{1}{2}\right)$. In fact, integrating by parts leads to

$$
\begin{aligned}
\int_{s}^{\infty} x f(x) \mathrm{d} x & =\int_{s}^{\infty} \sqrt{\frac{x}{2 \pi}} \mathrm{e}^{-\frac{x}{2}} \mathrm{~d} x \\
& =\sqrt{\frac{2 s}{\pi}} \mathrm{e}^{-\frac{s}{2}}+\int_{s}^{\infty} f(x) \mathrm{d} x,
\end{aligned}
$$

while the change of variable yields

$$
\begin{aligned}
\int_{s}^{\infty} f(x) \mathrm{d} x & =\int_{s}^{\infty} \sqrt{\frac{1}{2 \pi x}} \mathrm{e}^{-\frac{x}{2}} \mathrm{~d} x \\
& =\sqrt{\frac{2}{\pi s}} \mathrm{e}^{-\frac{s}{2}} \int_{0}^{\infty} \sqrt{\frac{s}{s+x}} \frac{1}{2} \mathrm{e}^{-\frac{x}{2}} \mathrm{~d} x .
\end{aligned}
$$

From (43) it is obvious that

$$
T^{2}=\mathbb{E}\left(X^{2} \mid X^{2}>t\right)>t
$$

and

$$
\left|\mathbb{E}\left(X^{2} \mid X^{2}>s_{1}\right)-\mathbb{E}\left(X^{2} \mid X^{2}>s_{2}\right)\right| \leq C\left|s_{1}-s_{2}\right|
$$

for some positive constant $C$ when $s_{1}, s_{2}$ is bounded away from zero. In particular, we have

$$
\begin{aligned}
\left|\mathbb{E} T_{k}^{2}-T^{2}\right| & =\left|\mathbb{E}\left(\mathbb{E} T_{k}^{2} \mid X_{(k+1)}^{2}\right)-T^{2}\right| \\
& =\left|\mathbb{E}\left(\mathbb{E} T_{k}^{2} \mid X_{(k+1)}^{2}\right)-\mathbb{E}\left(T_{k}^{2} \mid X_{(k+1)}^{2}=t\right)\right| \\
& \leq C \mathbb{E}\left|X_{(k+1)}^{2}-t\right| \\
& \leq C \sqrt{\frac{t}{k \log \frac{n}{k}}}
\end{aligned}
$$

where the last inequality follows from [2] and Lemma 2, This proves

$$
\left|\mathbb{E} T_{k}-T\right| \leq \frac{C \sqrt{t}}{T \sqrt{k \log \frac{n}{k}}}
$$

But (44) implies $T>\sqrt{t}$, thus

$$
\left|\mathbb{E} T_{k}-T\right| \leq \frac{C}{\sqrt{k \log \frac{n}{k}}} .
$$

The proof is concluded by invoking (39) to obtain

$$
\mathbb{P}\left(\left|T_{k}-T\right|>\frac{C}{\sqrt{k \log \frac{n}{k}}}+\varepsilon\right) \leq 2 \mathrm{e}^{-\frac{k \varepsilon^{2}}{2}}
$$




\section{Discussions}

In this section we discuss the extension of Theorem 1 to sub-Gaussian matrices and that of Proposition 2 to general distributions. Revisiting the proof of Theorem 11, one finds that there are two places where being Gaussian is critical:

1. Applying Proposition 2 .

2. Bounding $\sum_{i, j=2}^{N} v_{i}^{\prime} v_{j}^{\prime}\left\langle\mathbf{b}_{i}, \mathbf{b}_{j}\right\rangle$ by independence and Hanson-Wright inequality.

To resolve these issues, we first discuss the extension of Proposition 2 to non-Gaussian cases. It is evident that the use of concentration of measure in proving Proposition 2 is redundant: one may instead try to bound $T_{k}-T$ and $\mathbb{E} T_{k}-T$ by utilizing Lemma 2 only. The main difficulty arising in doing so is that (38) is no longer applicable and

$$
T_{k}^{2} \mid X_{(k+1)} \stackrel{(d)}{=} \frac{1}{k} \sum Y_{i}
$$

no longer holds for discontinuous distributions as in (42). However, if $F$ is close to chisquared distribution, hence has small jumps, it is still possible to control the deviation probability in (38) by $\delta$. As for (42), we show a simple trick that reduces the situation to continous case. Let $G$ be the generalized inverse function of $F$, defined by

$$
G(x)=\inf \{t: F(t) \geq x\}
$$

and let $U_{1}, \ldots, U_{n}$ be i.i.d. random variables following uniform distribution on $[0,1]$. Then

$$
\left(X_{(1)}, \ldots, X_{(n)}\right) \stackrel{(d)}{=}\left(G\left(U_{(1)}\right), \ldots, G\left(U_{(n)}\right)\right)
$$

Note that $G$ is monotonically increasing. Hence $G\left(U_{(k+1)}\right)=s$ is equivalent to $U_{(k+1)} \in$ $[\alpha, \beta]$ for some $0 \leq \alpha \leq \beta \leq 1$. Thus $T_{(k)}^{2} \mid\left\{X_{(k+1)}=s\right\}$ has the same distribution as $\sum G^{2}\left(U_{(i)}\right) \mid\left\{U_{(k+1)} \in[\alpha, \beta]\right\}$. Since the distribution of $U_{(i)}$ is continuous, what we desire follows from controlling $\sum G^{2}\left(U_{(i)}\right) \mid U_{(k+1)}$ by the same argument in Proposition 2 and integrating.

In a word, we have sketched a proof that: Proposition 2 holds with slight modification for distributions that are sufficiently close to chi-squared. By central limit theorem, the distribution of $x_{i}$ defined after (14) converges to Gaussian, and consequently the distribution of $x_{i}^{2}$ converges to $\chi_{1}^{2}$. This implies that Proposition 2 remains valid for sub-Gaussian matrices, hence resolving the issue 1) above. 
Issue 2) is more delicate and requires some modifications in the statement of Theorem 1 to generalize to sub-Gaussian matrices. To avoid unnecessary technicalities, we will not explicitly state these modifications here, but instead explain the main strategy for such generalization as follows. It follows from our construction that $\left(v_{2}^{\prime}, \ldots, v_{n}^{\prime}\right)$ is $(s-1)$-sparse, hence we have

$$
\frac{1}{n}\left|(n-1)\left(1-v_{1}^{2}\right)-\sum_{i, j=2}^{N} v_{i}^{\prime} v_{j}^{\prime}\left\langle\mathbf{b}_{i}, \mathbf{b}_{j}\right\rangle\right| \leq\left(2 \delta_{s-1}(\mathbf{B})+\delta_{s-1}^{2}(\mathbf{B})\right)\left(1-v_{1}^{2}\right),
$$

where

$$
\mathbf{B}=\frac{1}{\sqrt{n}}\left[\mathbf{b}_{2}, \ldots, \mathbf{b}_{N}\right] \in \mathbb{R}^{n \times(N-1)} .
$$

Note that $\mathbf{B}$ is a submatrix of $\mathbf{P A}$, where $\mathbf{P}$ is the orthogonal projection onto the orthogonal complement of $\mathbf{a}_{1}$. It follows from, for instance, Cauchy interlacing law that $\delta_{s-1}(\mathbf{B}) \leq \delta_{s}(\mathbf{A})$. For sub-Gaussian random matrices $\mathbf{A}$, upper bounds of $\delta_{s}(\mathbf{A})$ are wellknown (Theorem 2). Thus the term $\sum_{i, j=2}^{N} v_{i}^{\prime} v_{j}^{\prime}\left\langle\mathbf{b}_{i}, \mathbf{b}_{j}\right\rangle$ is of the same order as $T_{k}$ with overwhelming probability. To get rid of such weakness, one may take $v_{1}$ to be properly close to 1 , which yields faster decay of the term $\left(1-v_{1}^{2}\right) \delta_{s-1}(\mathbf{B})$ than the decay of $\sqrt{1-v_{1}^{2}} \sqrt{\sum_{j=1}^{s-1} x_{(j)}^{2}}$, so the error term will be eventually dominated by $\sqrt{1-v_{1}^{2}} \sqrt{\sum_{j=1}^{s-1} x_{(j)}^{2}}$. The error term this approach gives is actually worse than that in Theorem 1 by a constant, which should not be a serious concern in most applications, for instance, in Corollary 1 .

\section{Numerical Experiments}

We present some numerical calculations in this section to provide an intuition of our new lower and upper bounds for the RIP constant described in Theorem 1 and Proposition 1 . We fix sparsity levels $s / N=(0.1,0.01,0.001), N=10000$ and compute the $99 \%$-confidence intervals for different compression rates, see Figure 1, We find that when the compression rate $N / n$ is not too large, our new bounds are quite tight compared to previous ones.

\section{Conclusion}

In this paper we gave a lower bound of RIP constants for Gaussian random matrices and discussed the strategy to generalize this result to sub-Gaussian random matrices. For Gaussian random matrices, this bound was shown to be tight by comparing with a new upper bound 

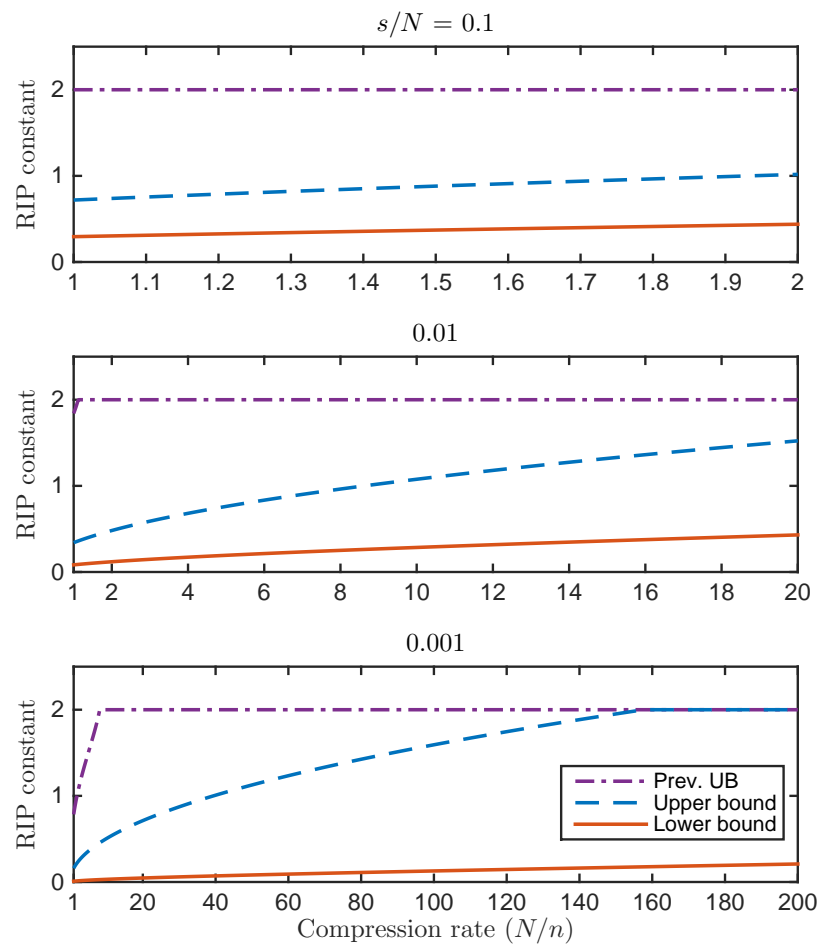

Figure 1: Plot of the lower bound and the upper bound of the RIP constant derived in this paper comparing with previous results. The $x$-coordinate is compression rate. The sparsity level $s / N$ is taken to be $0.1,0.01,0.001$ respectively in three figures. Parameters $N$ are fixed to 1000, and confidence level is 0.99. All curves are truncated above by 2 for visual convenience.

of RIP constants which is better than previous results by a multiplicative constants in most cases. The new lower bound of RIP constants implies the fact that the minimal number of sub-Gaussian measurements needed to enforce the RIP of order $s$ is $\Omega(s \log (\mathrm{e} N / s))$, which was proved in literature by with a much more sophisticated approach. In our proof we also established a concentration inequality for sum of top order statistics, which we believe to be of interest in other fields besides compressed sensing.

\section{Appendix: Proof of Proposition 3}

It is well-known that Gaussian tail bound is asymptotically equivalent to $\frac{2}{x} \mathrm{e}^{-x^{2} / 2}$ :

$$
\mathbb{P}(|X|>x) \sim \frac{2}{x} \mathrm{e}^{-x^{2} / 2}
$$


Thus

$$
\frac{s}{N} \sim \mathbb{P}\left(X^{2}>t\right) \sim \frac{2}{\sqrt{t}} \mathrm{e}^{-t / 2}
$$

Taking logarithms on both sides, we obtain

$$
t+\log t \sim 2 \log \frac{N}{s}
$$

As $s / N \rightarrow \infty, t$ also tends to infinity, thus $\log t=o(t)$. It follows that

$$
t \sim 2 \log \frac{N}{s}
$$

To prove $T \sim \sqrt{2 \log \frac{N}{s}}$, we will use (43). By bounded convergence theorem we see

$$
\lim _{s \rightarrow \infty} \mathbb{E} \sqrt{\frac{s}{s+Y}}=1
$$

This combined with (43) implies

$$
\mathbb{E}\left(X^{2} \mid X^{2}>t\right) \sim t
$$

hence

$$
T=\sqrt{\mathbb{E}\left(X^{2} \mid X^{2}>t\right)} \sim \sqrt{t} \sim \sqrt{2 \log \frac{N}{s}}
$$

\section{Appendix: Tools from Probability Theory}

In this appendix we collect some tools from probability theory that play a role (but are not essential) in our proof.

Theorem 4 (Concentration of measure, [10]) Let $X$ be a standard Gaussian vector taking values in $\mathbb{R}^{n}$ and $f: \mathbb{R}^{n} \rightarrow \mathbb{R}$ a K-Lipshitz function, i.e.

$$
|f(\mathbf{x})-f(\mathbf{y})| \leq K\|\mathbf{x}-\mathbf{y}\|, \quad \text { for all } \mathbf{x}, \mathbf{y} \in \mathbb{R}^{n}
$$

Then we have

$$
\begin{gathered}
\mathbb{P}(f(X)-\mathbb{E} f(X)>t) \leq \mathrm{e}^{-\frac{t^{2}}{2 K^{2}}}, \\
\mathbb{P}(f(X)-\mathbb{E} f(X)<-t) \leq \mathrm{e}^{-\frac{t^{2}}{2 K^{2}}} .
\end{gathered}
$$

The next lemma is on comparison of Gaussian processes that plays an important role in proving Proposition 1 , 
Lemma 3 (Slepian-Fernique lemma, [15]) Let $\left(X_{s, t}\right)_{s \in S, t \in T}$ and $\left(Y_{s, t}\right)_{s \in S, t \in T}$ be two Gaussian processes defined on the index set $S \times T$. Assume that for any $s, s^{\prime} \in S, t, t^{\prime} \in T$, $s \neq s^{\prime}$ we have

$$
\begin{gathered}
\mathbb{E}\left|X_{s, t}-X_{s, t^{\prime}}\right|^{2} \leq \mathbb{E}\left|Y_{s, t}-Y_{s, t^{\prime}}\right|^{2}, \\
\mathbb{E}\left|X_{s, t}-X_{s^{\prime}, t^{\prime}}\right|^{2} \geq \mathbb{E}\left|Y_{s, t}-Y_{s^{\prime}, t^{\prime}}\right|^{2} .
\end{gathered}
$$

Then

$$
\mathbb{E} \min _{s \in S} \max _{t \in T} X_{s, t} \leq \mathbb{E} \min _{s \in S} \max _{t \in T} Y_{s, t} .
$$

Corollary 4 Let $\left(X_{t}\right)_{t \in T}$ and $\left(Y_{t}\right)_{t \in T}$ be two Gaussian processes defined on the index set T. Assume that for any $t, t^{\prime} \in T$ we have

$$
\mathbb{E}\left|X_{t}-X_{t^{\prime}}\right|^{2} \leq \mathbb{E}\left|Y_{t}-Y_{t^{\prime}}\right|^{2}
$$

Then

$$
\mathbb{E} \max _{t \in T} X_{t} \leq \mathbb{E} \max _{t \in T} Y_{t}
$$

Proof of Lemma 1 We first check that

$$
\mathbb{E}\left|X_{\mathbf{u}, \mathbf{v}}-X_{\mathbf{u}^{\prime}, \mathbf{v}}\right|^{2}=\mathbb{E}\left|Y_{\mathbf{u}, \mathbf{v}}-Y_{\mathbf{u}^{\prime}, \mathbf{v}}\right|^{2}
$$

In fact, the left hand side is equal to $\left\|\mathbf{u}-\mathbf{u}^{\prime}\right\|^{2}\|\mathbf{v}\|^{2}$, while the right hand is equal to $\left\|\mathbf{u}-\mathbf{u}^{\prime}\right\|^{2}$. This implies (56) since $\|v\|=1$ by definition of $\Psi_{k}$.

Next we show

$$
\mathbb{E}\left|X_{\mathbf{u}, \mathbf{v}}-X_{\mathbf{u}^{\prime}, \mathbf{v}^{\prime}}\right|^{2} \leq \mathbb{E}\left|Y_{\mathbf{u}, \mathbf{v}}-Y_{\mathbf{u}^{\prime}, \mathbf{v}^{\prime}}\right|^{2},
$$

for all $\mathbf{u}, \mathbf{u}^{\prime} \in \mathbb{S}^{n-1}, \mathbf{v}, \mathbf{v}^{\prime} \in \Psi_{k}$. A little computation shows this is equivalent to

$$
\sum_{i} \sum_{j}\left|u_{i} v_{j}-u_{i}^{\prime} v_{j}^{\prime}\right|^{2} \leq\left\|\mathbf{u}-\mathbf{u}^{\prime}\right\|^{2}+\left\|\mathbf{v}-\mathbf{v}^{\prime}\right\|^{2}
$$

By expanding $\left|u_{i} v_{j}-u_{i}^{\prime} v_{j}^{\prime}\right|^{2}$ as

$$
\left(u_{i}-u_{i}^{\prime}\right)^{2} v_{j}^{2}+u_{i}^{\prime 2}\left(v_{j}-v_{j}^{\prime}\right)^{2}+2\left(u_{i}-u_{i}^{\prime}\right) v_{j} u_{i}^{\prime}\left(v_{j}-v_{j}^{\prime}\right),
$$

equation (57) is reduced to

$$
\left(1-\left\langle\mathbf{u}, \mathbf{u}^{\prime}\right\rangle\right)\left(1-\left\langle\mathbf{v}, \mathbf{v}^{\prime}\right\rangle\right) \geq 0
$$

which readily follows from Cauchy-Schwarz inequality.

The upper bound in Lemma 1 is an easy corollary of (57) and Corollary 4, while the lower bound follows from (56), (57) and Lemma 3 , 


\section{References}

[1] E. J. Candès, "The restricted isometry property and its implications for compressed sensing," Comptes Rendus Mathematique, vol. 346, no. 9-10, pp. 589-592, 2008.

[2] S. Boucheron, M. Thomas et al., "Concentration inequalities for order statistics," Electronic Communications in Probability, vol. 17, 2012.

[3] H. A. David and H. N. Nagaraja, "Order statistics," Encyclopedia of Statistical Sciences, 2004.

[4] H. Rauhut, J. Romberg, and J. A. Tropp, "Restricted isometries for partial random circulant matrices," Applied and Computational Harmonic Analysis, vol. 32, no. 2, pp. $242-254,2012$.

[5] S. Foucart and H. Rauhut, A Mathematical Introduction to Compressive Sensing. Springer Science \& Business Media, 2013.

[6] A. M. Tillmann and M. E. Pfetsch, "The computational complexity of the restricted isometry property, the nullspace property, and related concepts in compressed sensing," IEEE Transactions on Information Theory, vol. 60, no. 2, pp. 1248-1259, 2013.

[7] A. S. Bandeira, E. Dobriban, D. G. Mixon, and W. F. Sawin, "Certifying the restricted isometry property is hard," IEEE Transactions on Information Theory, vol. 59, no. 6, pp. 3448-3450, 2013.

[8] R. Baraniuk, M. Davenport, R. DeVore, and M. Wakin, "A simple proof of the restricted isometry property for random matrices," Constructive Approximation, vol. 28, no. 3, pp. 253-263, 2008.

[9] S. Foucart, A. Pajor, H. Rauhut, and T. Ullrich, "The gelfand widths of $\ell_{p}$-balls for $0<p \leq 1$," Journal of Complexity, vol. 26, no. 6, pp. 629-640, 2010.

[10] M. Ledoux, The concentration of measure phenomenon. American Mathematical Soc., 2001, no. 89 .

[11] P. Massart, "The tight constant in the Dvoretzky-Kiefer-Wolfowitz inequality," The Annals of Probability, pp. 1269-1283, 1990. 
[12] M. Rudelson, R. Vershynin et al., "Hanson-wright inequality and sub-gaussian concentration," Electronic Communications in Probability, vol. 18, 2013.

[13] F. Qi, Q.-M. Luo et al., "Bounds for the ratio of two gamma functions - from wendel's and related inequalities to logarithmically completely monotonic functions," Banach Journal of Mathematical Analysis, vol. 6, no. 2, pp. 132-158, 2012.

[14] T. M. Cover and J. A. Thomas, Elements of information theory. John Wiley \& Sons, 2012.

[15] Y. Gordon, "Elliptically contoured distributions," Probability Theory and Related Fields, vol. 76, no. 4, pp. 429-438, 1987. 